\title{
Posibilidades del Blockchain en Educación
}

\section{Blockchain possibilities in Education}

\author{
Antonio Bartolomé, Carles Lindín \\ Instituto de Investigación en Educación, Universitat de Barcelona, España \\ abartolomepina@gmail.com; carles.lindin@ub.edu \\ https://orcid.org/0000-0002-8096-8278; https://orcid.org/0000-0002-3640-1258
}

\section{Resumen}

Blockchain es una nueva tecnología que está irrumpiendo con fuerza en muchos campos, y también en el sistema educativo. Pero no se trata de una tecnología específica con la que se puede esperar mejorar una situación concreta: se trata más bien de una tecnología que permite registrar eventos de una forma totalmente innovadora. Y esto puede traducirse en cambios insospechados. Tras presentar qué es blockchain y algunos ejemplos de usos sociales, se analizan, en las aplicaciones actualmente en uso, los problemas a los que la tecnología pretende dar respuesta. Una revisión de las experiencias más destacadas, todas en una fase incipiente de desarrollo, nos lleva a algunas conclusiones críticas sobre su viabilidad.

\section{Palabra clave}

Blockchain; Criptomonedas; Bitcoin; Educación; Evaluación; Acreditación; Aprendizaje; Contratos inteligentes

\begin{abstract}
The blockchain is a new technology that is breaking through in many fields, and also in the educational system. But it is not a specific technology which you can expect to improve a concrete situation: It is more a technology that allows registering events from an innovative form. And this can be translated into unsuspected changes. After presenting what Blockchain is and some examples of social uses, in the applications currently in use, there is an analysis of the problems to which the technology aims to answer. A review of the most outstanding experiences, all in an early phase of development, leads us to some critical conclusions about its viability.
\end{abstract}

\section{Keywords}

Blockchain; Cryptocurrency; Bitcoin; Education; Evaluation; Accreditation; Learning; Smart contracts

\section{La tecnología}

Hace más de 30 años que convivimos con el "dinero digital" (por ejemplo, el sistema VISA o MASTERCARD), en que la información de las transacciones se gestiona desde un servidor central (Chaum, 1983). La idea era buena, pero parece que ha sido imposible compatibilizar la centralización de la información con el anonimato y evitar el fraude. Por eso no debe extrañar el éxito de las criptomonedas (monedas virtuales como el Bitcoin), que han podido hacer compatibles estos tres aspectos. La criptomoneda no utiliza un servidor central que registra todos los intercambios, sino que éstos son replicados en todos los ordenadores de los usuarios de la red, esto sí, convenientemente encriptados y de modo anónimo (Wright \& De Filippi, 2015). 
Para conseguirlo, se utiliza una tecnología llamada blockchain. Esta tecnología supone el cambio más importante en relación a las bases de datos desde que se desarrollaron hace casi 50 años. Puede describirse de forma breve así: si poseemos, por ejemplo, las calificaciones que obtiene un estudiante o los títulos académicos que consigue (lo que llamamos en lenguaje blockchain "un registro de eventos"), cada nuevo evento (cada nueva calificación o título académico), agrupado en un bloque con otros, es encriptado, firmado digitalmente utilizando un hash (una especie de huella digital encriptada) y encadenado (relacionado) con los bloques anteriores. De esta manera se construye la cadena de bloques (traducción de blockchain), que se guarda en los ordenadores de quienes utilizan la red.

Si cada usuario posee una copia de la cadena, no puede alterarla: sería necesario introducir el cambio en las copias de los otros usuarios al mismo tiempo. Además, se modificaría el hash con la firma electrónica y se detectaría el fraude. Así mismo, es necesaria la autorización del propietario del evento para acceder a la información y leerla, pues está encriptada. Por tanto, de un modo sorprendente blockchain puede hacer compatible el anonimato con la transparencia.

\subsection{Aplicaciones}

La fiabilidad del uso de blockchain en las criptomonedas ha llevado a diversas entidades financieras a tratar de incorporarla en sus sistemas: el sistema de las tarjetas de crédito VISA (Arnold, 2016), diversos bancos como el Santander, Deutsche Bank o el Banco de Inglaterra (Allison, 2015; Gallen, 2016). Pero no solo los bancos: de las transacciones comerciales también se benefician, por ejemplo, el comercio internacional (Morales, 2017), el registro de los derechos de autor (Behrens, 2018) o la transparencia en las finanzas públicas (Goswami, 2016).

Del ámbito financiero y comercial, blockchain ha pasado a ser aplicado en muchos otros campos de la actividad humana. En Sanidad se valora su oportunidad para guardar los registros de los pacientes de un modo seguro y al tiempo asequible a los diferentes actores del campo de la salud (Pérez, 2016). También se está aplicando para asegurar la identidad de las personas, especialmente aquellas que se encuentran en situación vulnerable como es el caso de los refugiados (Go, 2018), o creando monedas sociales para grupos o situaciones específicas (Jané, 2017). La Asociación Española de Fundaciones, que agrupa a más de 150 entidades, también va a utilizar blockchain, en este caso, para hacer un seguimiento de las donaciones (Blázquez, 2018). La lista sería interminable. Y de ahí no debe extrañar que lleguemos al campo educativo.

\subsection{Extensiones de la tecnología}

Blockchain se aplica en conjunción con otras tecnologías como puede ser bases de datos de documentos, por ejemplo, copias gráficas de certificaciones, portafolios digitales, o simples 
descripciones pormenorizadas. Los bloques incorporan registros relativamente poco voluminosos. Un bloque podría ocupar $1 \mathrm{Mb}$ y cada bloque incorpora varios registros.

En muchas situaciones esta tecnología se vincula a smart contracts (contratos inteligentes). En este caso un software contiene las cláusulas del contrato, almacenado de modo que se ejecuta automáticamente en cuanto se dan las condiciones establecidas. Ello permite ahorrar trabajo burocrático y costo.

También puede vincularse al Internet de las cosas. Por ejemplo, el contrato se ejecuta y el registro se crea en el momento que un paquete llega a su destino, detectado de modo automático.

Aunque Bitcoin es la plataforma de blockchain más conocida, en el ámbito educativo está ganando aceptación Ethereum. En cualquier caso, estas y otras plataformas están en constante evolución, tratando de solucionar problemas como por ejemplo el costo que supone la generación de los bloques.

Lo que está generando grandes expectativas y despierta el interés en una red como Openergy es la posibilidad de un sistema de registros abierto, privado y, a la vez, seguro. También la Ciencia abierta está considerando la posibilidad de utilizar blockchain como un sistema de registro de los procesos investigadores que garantice sus derechos al tiempo que abra los resultados a la colaboración. Blockchain se percibe en este y otros campos como lo que fue en su momento el lenguaje HTML y el protocolo HTTP, desarrollados por Berners-Lee inicialmente como un sistema que facilitara la comunicación entre investigadores, y que se ha convertido en la Web tal como hoy se conoce.

Pero al igual que las versiones primitivas de HTML y los primeros sitios web son hoy arqueología, en pocos años probablemente la tecnología va a evolucionar de modos que ni siquiera se puede hoy imaginar. En Educación recién se comienza a aplicar para resolver algunos problemas (Bartolomé y Moral, 2018).

\section{Dos grandes retos del sistema educativo}

La Educación en el siglo XXI se enfrenta a importantes retos que superan la mera optimización de los procesos de enseñanza-aprendizaje (Bartolomé, 2011; Bartolomé y Grané, 2013), son los cambios producidos en una sociedad por unas tecnologías para el conocimiento que, como señala el síndrome de Frankenstein (Postman, 1991), cambian no solo nuestras costumbres sino también nuestra manera de pensar.

La tecnología de las cadenas de bloques parece aportar una solución a dos problemas derivados de estos cambios, a dos grandes retos del sistema educativo: las limitaciones del actual sistema de acreditación y las limitaciones que genera su organización en grados y niveles. 


\subsection{La acreditación de las competencias y saberes}

Aprender ya no es una actividad que se realiza durante un periodo inicial de modo formal, y que se enriquece con la experiencia en la práctica profesional y vital. El aprendizaje a lo largo de la vida se ha convertido en una necesidad laboral (Longworth, 2005), acorde al modo de aprender humano (Bruer, 1999), un requerimiento de la ciudadanía del siglo XXI (Martín Ortega, 2008), que afecta tanto al marco social en que nos movemos, como al aprendizaje social biográfico (Alheit \& Dausien, 2002).

Aparecen otros sistemas alternativos de formación: boot camps especialmente en el campo informático (Smith \& Bickford, 2004), MOOC o cursos masivos abiertos y en línea (Dinevski \& Kokol, 2004; García-Peñalvo, Fidalgo-Blanco \& Sein-Echaluce, 2017, 2018), la clase del ancho mundo (Breslow et al., 2013), los vídeos de la Academia Khan (Thompson, 2011) o simplemente Youtube (Das, 2011), entre otros. Se señala la superación de la distinción entre educación formal por un lado y la educación informal y la no-formal por otro (La Belle, 1982; Tuijnman \& Boström, 2002).

También aparecen programas que serían considerados como parte de sistemas no-formales pero que recurren a las "insignias" (badges) dentro de un marco de aprendizaje basado en la gamificación (Sánchez i Peris, 2015) para incorporar elementos motivacionales (incentivadores) al aprendizaje. Estas insignias a modo de premios adquieren también valor acreditativo cambiando la perspectiva de esos programas (Abramovich, Schunn \& Higashi, 2013; Gibson, Ostashewski, Flintoff, Grant \& Knight, 2015) incluso en campos tan delicados como la formación médica (Mehta, Hull, Young \& Stoller, 2013).

Por otro lado, los empleadores solicitan información como el Curriculum Vitae (CV) u Hoja de Vida elaborado por el propio sujeto: estos documentos no siempre presentan garantías que acredite la veracidad de lo expuesto en ellos. Acompañarlos de los correspondientes certificados resulta oneroso y complejo de comprobar.

Los títulos académicos formales (grados, postgrados, doctorados) se muestran insuficientes para describir la capacidad y conocimientos de los sujetos, siendo necesario acompañaros de información complementaria como el "Suplemento europeo al título" que permita interpretar el auténtico significado de esos títulos.

Tradicionalmente la validez de una acreditación o un título ha sido proporcionada por una autoridad central. Esta realidad contrasta con la existencia de plataformas como Google Scholar, que proporciona información sobre los méritos de las publicaciones de un autor en base a las citas recibidas. Las redes sociales de investigación y los id estandarizados de investigadores son otra solución al problema de la identificación del CV en el ámbito académico: Orcid, ReserarchGate, Academia, Mendeley, Researcher 
ID... (García-Peñalvo, 2018). Aunque, de nuevo, no son infrecuentes los errores, incluso intencionados, introducidos por los propios sujetos.

¿Es posible un sistema de acreditación que dé fe de las competencias poseídas, garantizando que no han sido falseadas, y que al mismo tiempo preserve la privacidad de la información fuera de los momentos en que el ciudadano acepta proporcionarla a un posible empleador? Las cadenas de bloques permitirían acreditar los elementos de un CV elaborado por el usuario, impidiendo la manipulación o alteración de datos, diseminadas través de un sistema distribuido sin guardar los datos en un centro sujeto a ataques o violaciones de su integridad. Se trata de un cambio que tendrá un alto impacto en el sistema educativo, pero también que tardará más de 4 años para que comience a implantarse (Sharples et al., 2016).

Entre las primeras aplicaciones para solventar este problema utilizando blockchain se encuentra la Universidad de Nicosia, que certifica algunos títulos expedidos desdela facultad de Economía(Koulaidis, 2018), y el Media Lab del MIT. En el MIT comenzaron a distribuir certificados a los participantes en su programa de becas ajuntas a la dirección (Director's Fellows program), autentificados mediante la tecnología blockchain (Raths, 2016). Durante 2016 algunos foros se han hecho eco de las posibilidades de las cadenas de bloques en éste y otros campos. Devine (2015) lo define como una posible transferencia universal de créditos entre instituciones. Esto facilita el movimiento de los estudiantes entre instituciones, pero también tiene aplicación al facilitar a los empleadores información fidedigna sobre las acreditaciones de sus empleados, tanto las previas como las que se adquieren en el propio puesto de trabajo. El MIT Media Lab y Learning Machine han puesto en marcha Blockcerts, una infraestructura abierta para credenciales académicas basadas en blockchain.

\subsection{La individualización del itinerario de aprendizaje}

Con la aparición de la escuela pública del siglo XIX, se produce una irrupción masiva de nuevos sujetos a un sistema educativo: podemos atender a las necesidades y capacidades individuales de cada sujeto, pero a un costo inasumible si debe basarse en una ratio 1:1 (un educador por estudiante). Así aparecen las agrupaciones por grados y la estructura actual del sistema educativo, basado en una estructuración ligada al reconocimiento acreditativo. Pero ese reconocimiento se basa en la aplicación de los mismos criterios, objetivos y metodologías a todos los sujetos, en función del nivel en que se encuentran.

Pero los alumnos son diferentes: tienen diferentes necesidades, diferentes intereses, diferentes habilidades y diferentes formas/estilos de aprender. Son muchas las iniciativas y teorías que han buscado atender a esas diferencias, muchas veces con la ayuda de la tecnología: enseñanza programada, máquinas de enseñar de Skinner (1960), educación personalizada, escuela sin grados, EAO (enseñanza asistida por ordenador), sistemas inteligentes deEAO, tutoriales, tutoriales inteligentes, 
agentes docentes inteligentes... hasta el aprendizaje adaptativo actual basado en la minería de datos.

Las necesidades relacionadas con la formación a lo largo de la vida están también convirtiendo el sistema de agrupación por clases y estratificación por grados en una solución excesivamente onerosa. No debe extrañar el interés despertado por soluciones como el aprendizaje adaptativo.

Blockchain se configura como una tecnología que permite al estudiante ir eligiendo sus paquetes de aprendizaje de entre una oferta variada, guiado por el formador y con el soporte de programas de valoración y guía, gestionando contratos inteligentes (smart contracts), generando un registro de seguimiento de sus aprendizajes.

En este sentido, una noticia de Sony fechada el 22 de febrero de 2016 habla del desarrollo de una tecnología basada en blockchain por Sony Global Education, que permitiría tanto compartir los resultados académicos como registrar los progresos en el aprendizaje.

Asimismo, OpenBlockchain es un proyecto de la Open University (UK) con menos de un año de vida y que plantea el uso de blockchain tanto como una respuesta a la realidad de un nuevo ecosistema de aprendizaje como para la gestión de un aprendizaje individualizado en el que los estudiantes deben mantener un registro de las actividades realizadas y los logros obtenidos (Sharples y Domingue, 2018).

Estos dos retos responden a estos cuatro problemas:

- La falsificación de las titulaciones académicas.

- La entrada en el mercado de nuevos operadores educativos.

- $\quad$ La sociedad que aprende: educación formal vs. no formal.

- La individualización de los aprendizajes.

\section{Proyectos en el marco del sistema educativo}

Podemos ver cómo las primeras aplicaciones en el sistema educativo han respondido a estos problemas.

\subsection{La falsificación de las titulaciones académicas}

Quizás, la primera aplicación de blockchain fue la ya citada: la Universidad de Nicosia decidió utilizar esta tecnología para asegurar la validez de los certificados que emitía en algunos programas formativos (Koulaidis, 2018). 
Lo que la universidad aseguraba no era únicamente que el certificado no podía ser falsificado, lo que también podría obtenerse aplicando una firma digital a un fichero acreditativo, sino también que el acceso a esta certificación fuese inmediato y no dependiese de que el estudiante conservara copia de esos ficheros. Además, comenzaba a generar un nuevo sistema de organización del currículo ligado a los certificados emitidos.

Han sido varios los desarrollos posteriores. La Dubai Future Foundation, entre otros, ha dado apoyo al proyecto Educhain, que comenzó incorporando a 15 instituciones interesadas en participar en este proyecto (Abdul, 2018).

TrustED es una empresa que ofrece a las instituciones el uso de su plataforma (en fase alfa de desarrollo) para acreditar certificaciones de los estudiantes (TrustED Blockchain Ecosystem, 2018). Profesores, estudiantes o empleadores pueden registrarse en la plataforma y hacer uso de esta versión elemental de certificación vía blockchain.

Esta aplicación resulta interesante especialmente en países en los que no existe una regulación férrea de las titulaciones. En países en los que la expedición de títulos está sometida a un control central desde el gobierno también puede aportar nuevos estándares de agilidad y simplicidad al reconocimiento académico.

\subsection{La entrada en el mercado de nuevos operadores educativos}

La propuesta del MIT va más allá de la mera certificación. En realidad, trata de responder a la posibilidad de que nuevos operadores puedan entrar en el mercado de la formación (Schmidt, 2016). En su web (http://www.blockcerts.org/guide/) explica qué pretende y cómo participar. Se trata de crear una plataforma y unos estándares que permitan a las instituciones implementar blockchain en programas educativos.

El Blockcert incluye cuatro componentes:

- Distribuidor: la universidad o institución o empresa que crea certificados digitales conteniendo un amplio rango de afirmaciones sobre las destrezas, logros o características del estudiante, todo registrado en una cadena de bloques.

- Certificado: estos deben ajustarse a los requisitos de Open Badges (Fundación Mozilla).

- Verificador: alguien que sin necesidad de depender del "distribuidor" verifica que el certificado no ha sido alterado, que lo ha emitido una determinada institución y corresponde a un determinado individuo. 
- Cartera: cada estudiante almacena sus certificados en esta "cartera" compartiéndolos con otros, por ejemplo, con los empleadores.

Algunos ejemplos de la aplicación de Blockcerts:

- El propio Media Lab proporciona certificados digitales utilizando esta tecnología a los alumnos de su curso "Lab's 30th anniversay" en octubre de 2015.

- Learning Machine ha proporcionado certificados en Recursos Humanos a su personal.

- El campo de trabajo Emprendeduría Global (Global Entrepreneurship Bootcamp) entregó certificados de este tipo a los estudiantes que participaron en Seúl, marzo de 2016.

- El Laboratorio para la Ciudad hizo lo mismo con los participantes en un taller en México, en septiembre de 2015.

Varios centros de formación, valorados socialmente, pero sin un reconocimiento institucional, encuentran en blockchain la vía a una mayor presencia. The Holberton School es un centro de formación en ingeniería de software que se presenta como una universidad alternativa para la próxima generación de desarrolladores informáticos.

Uno de los proyectos más ambiciosos es the Woolf University que va más lejos generando un nuevo tipo de currículo individualizado.

\subsection{La sociedad que aprende: educación formal vs. no formal}

El reconocimiento de los aprendizajes realizados en entornos formales, pero fuera del sistema institucional lleva al reconocimiento de aprendizajes realizados en entornos no formales (Griffiths \& García-Peñalvo, 2016; García-Peñalvo \& Griffiths, 2015; García-Peñalvo, Griffiths, Jonhson, Sharples \& Sherlock, 2014). Esta línea de trabajo apenas se insinúa tímidamente. Pero parece una consecuencia bastante probable en un plazo medio-largo. Será un cambio radical en el modo como vemos la educación, institucionalizada y sistematizada desde el siglo XIX, que puede transformarse en el próximo siglo en algo muy diferente de lo que hoy se conoce por sistema educativo. Blockchain posibilitaría dar fe de la adquisición de aprendizajes adquiridos en diversidad entornos.

\subsection{La individualización de los aprendizajes}

The Knowledge Media Institute (Open University, Reino Unido) desarrolla el proyecto OpenBlockchain. Se trata de un proyecto más reflexionado que el del MIT, encaminado a una reformulación de la personalización de los aprendizajes. Especula con un futuro basado en blockchain a través de su plataforma Learning is Earning 2026 (http://www.learningisearning2026.org). 
El diseño de la plataforma fragmenta el contenido en pequeños bloques (lecciones...) que el estudiante realiza en el orden que desea. Cada unidad incorpora un contrato inteligente (smart contract) que se resolverá cuando el sujeto haya realizado los aprendizajes satisfactoriamente.

Devine (2015) nos habla de cómo la trayectoria del estudiante (y del profesor) se presenta de un modo transparente, y favorece por ejemplo la movilidad de los estudiantes al ofrecerles un sistema abierto y verificable de mostrar sus logros académicos en forma de "moneda" o unidad de intercambio. Devine también pone el ejemplo del reconocimiento de aprendizajes en una red entre pares (peer-to-peer learning network), mencionando la reducción de costos.

En la misma línea, el proyecto Edublocs, ligado al entorno de la red Openergy, organiza una materia impartida por varios profesores en varios grupos, en un conjunto de actividades que el estudiante realiza siguiendo su propio camino. La gestión de las actividades realizadas y sus logros se convierte en fiable mediante registros blockchain (Bartolomé, Lindín y Rivera, 2018).

\section{Discusión}

Blockchain es una tecnología básica que permite la gestión de bases de datos de eventos mediante un sistema no centralizado, replicado, de registros agrupados en bloques y encadenados mediante hash, proporcionando seguridad y accesibilidad a la información contenida.

De su primitiva aplicación al campo financiero y económico, se puede ver cómo en muy pocos años está invadiendo muchos ámbitos diferentes, y entre ellos, ha entrado en el sistema educativo como un modo de responder a dos retos importantes: la certificación académica y la estructuración en grados. Sin embargo, todo hace pensar que muy rápidamente van a aparecer otras aplicaciones insospechadas.

La discusión se plantea hoy en relación a esas aplicaciones y a los retos que pretenden afrontar. Desde el primer momento algunos autores han expresado sus dudas sobre el uso de blockchain, ya que lo vinculan con una visión mercantilista del "mercado" de la formación, como una vía para abrir ese mercado a nuevos "operadores".

Los blockchain parece que podrían abocar el sistema educativo a uno de estos dos escenarios, en su interpretación más extrema:

- Un sistema educativo totalmente abierto en el que todos pueden actuar como proveedores o como receptores de formación.

- Un sistema educativo cerrado en manos de grandes corporaciones que pasan a controlarlo sustituyendo a las instituciones actuales. 
Existen otros aspectos que plantean dudas sobre la viabilidad del uso de esta tecnología. No es de implementación sencilla e inmediata por motivos tecnológicos y económicos. Por otro lado, puede llevar a consecuencias no aceptables según la dirección en que se trabaje o la intencionalidad con que se haga.

Plantea retos en áreas como la privacidad, la transparencia, la función y el valor de las certificaciones e instituciones "oficiales". Finalmente, inquieta a unos ciudadanos que han visto en estos años cómo algunos cambios tecnológicos generan otros cambios no siempre deseables.

\section{Referencias}

Abdul, L. (2018). Dubai Home to the biggest Educational Blockchain Implementation Project. Unlock, 5/3/2018. https://goo.gl/Qs4rvq

Abramovich, S., Schunn, C. \& Higashi, R. M. (2013). Are badges useful in education?: it depends upon the type of badge and expertise of learner. Educational Technology Research and Development, 61, 217-232. doi: https://doi.org/10.1007/s11423-013-9289-2

Alheit, P. \& Dausien, B. (2002). The 'double face'of lifelong learning: Two analytical perspectives on a 'silent revolution'. Studies in the Education of Adults, 34(1), 3-22. doi: https://doi.org/10.1080/0266083 0.2002 .11661458

Allison, I. (2015). Bank of England: Central banks looking at 'hybrid systems' using Bitcoin's blockchain technology. International Business Time. https://goo.gl/YE4Gmb

Arnold, M. (2016). Visa invita a las entidades a probar su nuevo sistema de pagos bancarios basado en la tecnología del 'bitcoin'. Expansion (15/9/2016). https://goo.gl/yNYwyJ

Bartolomé, A. (2011). Comunicación y aprendizaje en la Sociedad del Conocimiento. Virtualidad, Educación y Ciencia, 2(2), 9-46. https://goo.gl/4hijLb

Bartolomé, A. \& Grané, M. (2013). Interrogantes educativos desde la sociedad del conocimiento. Aloma. Revista de Psicologia, Ciències de l'Educació i de l'Esport, 31(1), 73-82. https://goo.gl/ixiUEV

Bartolomé, A. \& Moral-Ferrer, J. M. (2018). Blockchain en Educación. Cadenas rompiendo moldes. Barcelona: LMI. Colección Transmedia XXI. https://goo.gl/8WtCoc

Bartolomé, A., Lindín, C. \& Rivera-Vargas, P. (2018). Gestión de programas de aprendizaje personalizado mediante cadenas de bloque. En A. Bartolomé y J.M. Moral-Ferrer: Blockchain en Educación. Cadenas rompiendo moldes, pp. 141-182. Barcelona: LMI. Colección Transmedia XXI. 
Behrens, A. (2018). BerkleelCE's Open Music Initiative to Host Music and Rights Management Blockchain Events. Blockchain News, 9/2/2018. https://goo.gl/CmnqLS

Blázquez, S. (2018). El Giving Tuesday 2018 certificará las donaciones con Blockchain. Blockchain Economía, 5/10/2018. https://goo.gl/B7BfNv

Breslow, L., Pritchard, D. E., DeBoer, J., Stump, G. S., Ho, A. D. \& Seaton, D. T. (2013). Studying learning in the worldwide classroom: Research into edX's first MOOC. Research \& Practice in Assessment, 8. https://goo.gl/GgZU17

Bruer, J. T. (1999). The myth of the first three years: A new understanding of early brain development and lifelong learning. New York: The Free Press.

Chaum, D. (1983). Blind signatures for untraceable payments. In Chaum, D., Rivest, R.L. \& Sherman, A.T. (eds), Advances in Cryptology. (pp.199-203). New York: Springer. doi: https://doi.org/10.1007/9781-4757-0602-4_18

Das, A. K. (2011). Emergence of open educational resources (OER) in India and its impact on lifelong learning. Library Hi Tech News, 28(5), 10-15. doi: https://doi.org/10.1108/07419051111163848

Devine, P.M. (2015). Blockchain learning: can crypto-currency methods be appropriated to enhance online learning? In Proceedings of ALT Online Winter Conference 2015, 7-10 December 2015. http://oro. open.ac.uk/44966/

Dinevski, D. \& Kokol, P. (2004). ICT and lifelong learning. European Journal of Open, Distance and E-Learning, 7(2). https://goo.gl/soXgZx

García-Peñalvo,F.J.,Griffiths,D.,Jonhson,M.,Sharples,P.\&Sherlock,D.(2014). Problemsandopportunities in the use of technology to manage informal learning. In F. J. García-Peñalvo (Ed.), Proceedings of the Second International Conference on Technological Ecosystems for Enhancing Multiculturality (TEEM'14) (pp. 573-580). New York, USA: ACM. doi: https://doi.org/10.1145/2669711.2669958

García-Peñalvo, F. J. \& Griffiths, D. (2015). Rethinking informal learning. In G. R. Alves \& M. C. Felgueiras (Eds.), Proceedings of the Third International Conference on Technological Ecosystems for Enhancing Multiculturality (TEEM'15) (Porto, Portugal, October 7-9, 2015) (pp. 457-459). New York, USA: ACM. doi: https://doi.org/10.1145/2808580.2808648

García-Peñalvo, F. J., Fidalgo-Blanco, Á. \& Sein-Echaluce, M. L. (2017). Los MOOC: Un análisis desde una perspectiva de la innovación institucional universitaria. La Cuestión Universitaria, 9, 117-135.

García-Peñalvo, F. J. (2018). Identidad digital como investigadores. La evidencia y la transparencia de 
la producción científica. Education in the Knowledge Society, 19(2), 7-28. doi: https://doi.org/10.14201/ eks2018192728

García-Peñalvo, F. J., Fidalgo-Blanco, Á. \& Sein-Echaluce, M. L. (2018). An adaptive hybrid MOOC model: Disrupting the MOOC concept in higher education. Telematics and Informatics, 35, 1018-1030. doi: https://doi.org/10.1016/j.tele.2017.09.012

Gibson, D., Ostashewski, N., Flintoff, K., Grant, S. \& Knight, E. (2015). Digital badges in education. Education and Information Technologies, 20(2), 403-410. doi: https://doi.org/10.1007/s10639-0139291-7

Go, C. (2018). Blockchain and Identity Technologies. Medium 6/3/2018. https://goo.gl/rz9X2Y

Goswami, D (2016). Unchaining Blockchain: The Ultimate Transparency Tool? Blog of Government Innovators Network, Harvard Kennedy School, Ash Center for Democratic Governance and Innovation. (8/6/2016). https://goo.gl/f6prab

Griffiths, D. \& García-Peñalvo, F. J. (2016). Informal learning recognition and management. Computers in Human Behavior, 55A, 501-503. doi: https://doi.org/10.1016/j.chb.2015.10.019

Jané, C. (2017). Barcelona tendrá su moneda digital para uso social. El periódico, 14/11/2017. https:// www.elperiodico.com/es/barcelona/20171114/barcelona-tendra-su-moneda-digital-para-usosocial-6423549

Koulaidis, V. (2018). La tecnología Blockchain como herramienta pedagógica. En A. Bartolomé \& J.M. Moral-Ferrer: Blockchain en Educación. Cadenas rompiendo moldes, pp. 81-86. Barcelona: LMI. Colección Transmedia XXI.

La Belle, T. J. (1982). Formal, nonformal and informal education: A holistic perspective on lifelong learning. International Review of Education, 28(2), 159-175. doi: https://doi.org/10.1007/BF00598444

Longworth. N. (2005). El aprendizaje a lo largo de la vida en la práctica. Transformar la educación en el siglo XXI. Barcelona: Paidós.

Martín Ortega, E. (2008). Aprender a aprender: clave para el aprendizaje a lo largo de la vida. EstudiosInvestigaciones, 72.

Mehta, N. B., Hull, A. L., Young, J. B. \& Stoller, J. K. (2013). Just imagine: new paradigms for medical education. Academic Medicine, 88(10), 1418-1423. doi: https://doi.org/10.1097/ ACM.0b013e3182a36a07

Morales, T. (2017). Cadena de bloques. Cómo vender 25 toneladas de atún en 140 minutos gracias a 
blockchain. Retina. El Pais Economía (27/11/2017). https://goo.gl/KR28My

Pérez, I. (2016). Así puede ser el futuro del Blockchain en la salud según las grandes empresas. Criptonoticias (29/8/2016). https://goo.gl/vuvr5T

Postman, N. (1983). The Disappearance of Childhood. London: W H Allen.

Raths, D. (2016). How Blockchain Will Disrupt the Higher Education Transcript. Campus technology (16/05/2016). http://oro.open.ac.uk/44966/

Sánchez i Peris, F. J. (2015). Gamificación. Education in the Knowledge Society, 16(2), 13-15. doi:https:// doi.org/10.14201/eks20151621315

Schmidt, P. (2016). Blockcerts - An Open Infrastructure for Academic Credentials on the Blockchain. MLLearning (24/10/2016).

Sharples, M., de Roock, R., Ferguson, R., Gaved, M., Herodotou, C., Koh, E., Kukulska-Hulme, A., Looi, C-K, McAndrew, P., Rienties, B., Weller, M. \& Wong, L. H. (2016). Innovating Pedagogy 2016: Open University Innovation Report 5. Milton Keynes: The Open University. http://www.open.ac.uk/blogs/innovating/

Sharples, M. \& Domingue, J. (2018). Registro, reputación y recompensas en educación, basados en Blockchains. En A. Bartolomé \& J. M. Moral-Ferrer: Blockchain en Educación. Cadenas rompiendo moldes, pp. 107-118. Barcelona: LMI. Colección Transmedia XXI.

Skinner, B.F. (1960). Teaching Machines. The Review of Economics and Statistics, 42(3), 189-191. doi: https://doi.org/10.2307/1926170

Smith, K., \& Bickford, C. J. (2004). Lifelong learning, professional development, and informatics certification. Computers Informatics Nursing, 22(3), 172-178. doi: https://doi.org/10.1097/00024665200405000-00013

Thompson, C. (2011). How Khan Academy is changing the rules of education. Wired Magazine, 126, 1-5. https://goo.gl/PSR2Rq

TrustED Blockchain Ecosystem (2018). TrustED presents the platform's alfa release. Medium, 7/11/2018. https://goo.gl/yZpSpQ

Tuijnman, A. \& Boström, A. K. (2002). Changing notions of lifelong education and lifelong learning. International Review of Education, 48(1-2), 93-110. doi: https://doi.org/10.1023/A:1015601909731

Wright, A. \& De Filippi, P. (2015). Decentralized Blockchain Technology and the Rise of Lex Cryptographia. doi: https://doi.org/10.2139/ssrn.2580664 